\title{
Systemic lupus erythematosus and critical illness polyneuropathy
}

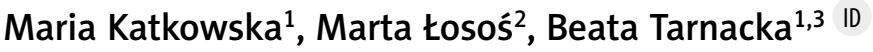 \\ ${ }^{1}$ Rehabilitation Clinic, National Institute of Geriatrics, Rheumatology and Rehabilitation, Warsaw, Poland \\ 2Early Arthritis Clinic, National Institute of Geriatrics, Rheumatology and Rehabilitation, Warsaw, Poland \\ ${ }^{3}$ Department of Rehabilitation, First Faculty of Medicine, Medical University of Warsaw, Warsaw, Poland
}

\begin{abstract}
Systemic lupus erythematosus (SLE) is a chronic disease of connective tissue with multi-organ involvement. Manifestation in the nervous system is one of the most difficult symptoms to assess and interpret. The aim of the study is to indicate diagnostic problems in patients with SLE in whom neurological symptoms are present at the time of diagnosis of SLE but also with complications that occurred after diagnosis.

In the presented case, the appearance of flaccid tetraparesis with areflexia suggested peripheral damage to the nervous system. In the electromyography performed in this patient, acute axonal polyneuropathy was mainly suspected. Further differential diagnosis should consider other acute and subacute developing polyneuropathies. Guillain-Barré polyneuropathy deserves special attention.

To our knowledge, this is the first case documented in the literature of the coexistence of critical illness polyneuropathy and SLE.
\end{abstract}

Key words: polyneuropathy, peripheral neuropathy, systematic lupus erythematosus, critical illness neuropathy.

\section{Introduction}

Systemic lupus erythematosus (SLE) is a chronic disease of connective tissue with multi-organ involvement. The worldwide prevalence of SLE is estimated at 130/100,000 inhabitants; it is more common in females, children and African Americans [1, 2]. In SLE, nervous system involvement is one of the most difficult symptoms to assess and interpret. Changes in the central nervous system are most often described as foci of vascular origin, and the least frequently as damage to the peripheral nervous system. In accordance with the findings of the American College of Rheumatology (ACR), in 1999 the nomenclature of neurological complications in SLE was unified and published [1, 2]. Neurological complications in SLE were reported in 14-95\% of cases [1, 3]. Such large differences in the frequency of diagnosing neurological involvement in SLE can be explained by difficulties in the diagnosis and clarification of these changes. Confirmation of nervous system involvement corresponds to a more severe course and mortality of this chronic autoimmune disease [4].

Ischemic strokes occur in 3-20\% of patients with SLE within the first 5 years of diagnosis [2]. The onset of illness is sudden with rapidly developing focal disorders and confirmation of diagnosis is obtained in neuroimaging tests. The mechanism of brain stroke in SLE can be various. Especially the increased clotting tendency in the course of antiphospholipid syndrome, embolism in the course of Liebman-Sacks endocarditis, lesions in the small cerebral vessels and increased occurrence of arteriosclerosis among patients with SLE underlie damage of brain structures.

Recurrent febrile conditions responding to antibiotic therapy often make it difficult to diagnose SLE and direct the diagnosis to infection. On the other hand, a severe infection can also cause nervous system changes and symptoms.

Importantly, nervous system symptoms occurring simultaneously with SLE symptoms can be brought to the fore and "mask" systemic disease. In this article, special 
attention is paid to critical illness polyneuropathy (CIP) as the cause of neurological changes in a patient with SLE and infection. Also the importance of rehabilitation in such cases is highlighted.

\section{Methods of literature review}

Literature from the last 10 years was taken into account; the search was carried out in the Web of Science and Medline databases by PubMed. The articles were qualified for analysis based on the following combination of key words: systematic lupus erythematosus, neuropathy and critical illness polyneuropathy.

The criterion excluding an article from further analysis was the lack of coexistence of CIP with SLE or the presence of neurological signs of CIP without association with SLE. Both original articles and case reports were taken into account. In searching the databases among 116 articles which were considered at screening the combination of SLE and CIP was not available.

In the present article the case of an SLE patient complicated with polyneuropathy fulfilling the criteria of CIP is described as the basis for further discussion and problem analysis.

\section{Results \\ Case description}

A 66-year-old female patient with recurrent fever and with a history of Hashimoto's disease and pulmonary embolism was referred to the rheumatology department with suspected connective tissue disease. Previously, this patient had been hospitalized several times in the city hospital, where despite applied antibiotic therapy (confirmed pneumonia and urinary tract inflammation) fevers up to $38.5^{\circ} \mathrm{C}$ persisted. Infections such us human immunodeficiency virus, hepatitis C virus, hepatitis C virus, cytomegalovirus, toxoplasmosis, Lyme disease, and tuberculosis were excluded. Afterwards bone marrow biopsy was also taken and there was no evidence of proliferative disease.

The diagnosis of SLE was confirmed according to the European Alliance of Associations for Rheumatology (EULAR)/ACR 2018 classification criteria (fever, fluid in both pleural cavities, leukopenia, thrombocytopenia, reduced components of $\mathrm{C} 3$ and $\mathrm{C} 4$ complement, high positive dsDNA, current anti-cardiolipin antibodies in both - IgM and IgG - classes of immunoglobulins and presence of anti- $\beta 2 \mathrm{GPI} 1$ antibodies).

Treatment with methylprednisolone $80 \mathrm{mg} /$ day was initiated. On the second day of hospitalization due to shortness of breath, progressive decrease in blood pressure, and elevated procalcitonin serum concentration
(3.61 ng/ml; $\geq 0.5$ increased risk of sepsis), the patient was transferred to the Intensive Care Unit, where the diagnosis of severe infection with risk of septic shock was established. Empirical antibiotic treatment (blood and urine cultures were negative) and glucocorticosteroid (GC) therapy were applied (methylprednisolone $80 \mathrm{mg}$, then $60 \mathrm{mg}$ ) and due to an episode of atrial fibrillation, amiodarone was used to achieve a sinus rhythm.

The patient after improving the general condition was re-hospitalized in the rheumatology department where GC therapy was continued and infusions of immunoglobulins in a total dose of $135 \mathrm{~g}$ were used to further improve the general condition. In the neurological picture, flaccid four-limb weakness persisted (dominant weakness of the left upper limb and right lower limb gradually increased), and no abnormalities were found in the brain imaging by magnetic resonance imaging. An episode of unconsciousness with left eye turning and hypotension but with rapid recovery prompted the performance of a series of tests including transthoracic echocardiography and computed tomography of coronary arteries, but no significant deviations were found. Electroencephalography recording did not reveal any irritation changes typical for epilepsy. Electromyography (EMG) showed features of generalized axonal damage of peripheral nerve motor fibers, features of $F$ wave disturbance from the right median nerve and limit values of conduction in sensory fibers of the right ulnar nerve. In addition, recording from the muscles of the upper limbs and lower limbs with features of depletion of exercise recording and an increased percentage of polyphasia. Critical illness polyneuropathy was suspected in the course of connective tissue disease and infection of unknown etiology.

In the follow-up EMG performed four weeks later, intensification of the lesions with a tendency to acute axonal polyneuropathy was observed, with a significant predominance of lesions in the nerves of the lower extremities with features of acute denervation.

The treatment with GCs was continued, methotrexate in the dose $15 \mathrm{mg}$ /week was initiated, and it was planned to include hydroxychloroquine after ophthalmic contraindications was excluded. The patient was receiving low molecular weight heparin in therapeutic doses due to a history of pulmonary embolism in the course of infection, and a maintenance dose during recovery. After achieving possible improvement and a stable condition the patient was transferred to the department of rehabilitation. Then in the neurological evaluation there was described generalized weakness of trunk and limb muscle strength, which was assessed as tetraparesis with pronounced dominance of the left upper limb and global paresis of the lower limbs with the dominance of 
the right and abolition of tendon reflexes in the limbs. During further hospitalization, persistent lower limb allodynia with a good response to treatment with pregabalin was found.

The improvement rehabilitation program consisted of: comprehensive improvement aimed at improving self-care and developing an independent transfer. Gradual verticalization, increase of muscular strength in relief, through isometric exercises, learning to change position in the bed, and coordination exercises were used. Also a gradual improvement in muscle strength and functional status was achieved. After 6 weeks of rehabilitation the patient changed positions in bed independently and was able to cover a distance of several meters with a walker with the assistance of therapists. The next cycle of rehabilitation after 3 weeks brought a result in the form of gait improvement; initially with a high support, the patient was taught to walk up the stairs, the technique of rising after a fall, and the muscular strength and range of mobility of the left shoulder were strengthened. Significant functional improvement was noted.

At discharge, the patient walked alone for short distances (about $50 \mathrm{~m}$ ), and for longer distances with a two-wheel support. The Barthel Index of Activities of Daily Living corresponds to the patient's current level of ability. The variables of the Barthel Index are shown in Figure 1.

\section{Discussion}

No similar case of SLE and CIP was found in the available literature. The difficulty in neurological diagnostics in the described patient was undoubtedly the occurrence of flaccid paresis, which was not accompanied by signs of central nervous system damage. No cranial nerve damage, or pathological symptoms and spasticity were found. Venous stroke is reported in $0.4-6 \%$ of SLE cases [5]. However, the dramatic course with characteristic headaches accompanied by convulsions and disturbances of consciousness also does not correspond to the present case.

In the described case, the appearance of flaccid tetraparesis with areflexia and transient persistent allodynia of the lower limbs, without disturbances in deep and superficial sensation (including feelings of discrimination, temperature, vibration) may suggest peripheral damage to the nervous system. In the performed EMG, the suspicion of acute axonal polyneuropathy was mainly confirmed. Neuropathy in SLE is reported in 3.4-7.5\% [2] and even $26 \%$ of cases [4]. The most common are sensory-motor and sensory polyneuropathy. It can be either axonal, demyelinating or mixed. SLE-dependent polyneuropathy manifests early and is associated with high

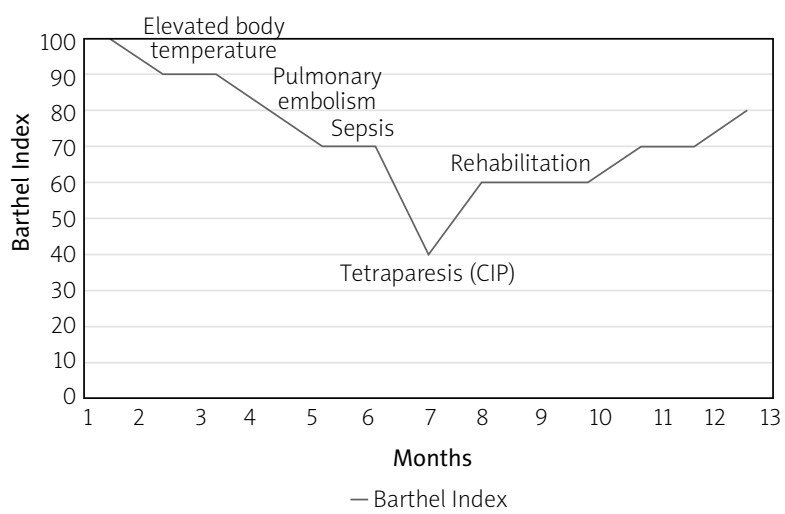

Fig. 1. Disability measured by Barthel Index during patient supervision

lupus activity. In our case, the symptoms of the disease occurred at its later stages, after the onset of infection.

Further differential diagnosis should consider other acute and subacute developing polyneuropathies in the course of infectious diseases including different serious infections, metabolic disorders, e.g. porphyria, uremia and diabetes, as well as toxic, druginduced and deficiency states including vitamin B12 deficiency [5]. However, the additional tests carried out in the described case did not indicate the above causes.

In the differential diagnosis of flaccid paresis, as was the case in the described patient, Guillain-Barré polyneuropathy (GBS) deserves special attention. It often has a more rapid course, with symptoms developing within a few days, and lasting about 4 weeks [6]. This is inflammatory polyneuropathy, in the vast majority of cases demyelinating. There is the acute inflammatory demyelinating polyradiculoneuropathy form, where acute demyelination occurs with possible secondary axonal degeneration, which can occur with a more severe course and longer duration, and the axonal forms described below. In two-thirds of cases, the disease is preceded by an infection of the respiratory or digestive tract 1 to 3 weeks before the onset of paresis. They develop rather symmetrically, which also did not happen with our patient. Usually, paresis is ascending, starting in the muscles of the legs, followed by the trunk, upper limbs and cranial muscles, especially facial muscles. Distal limb paresthesia often occurs.

Our patient also suffered from paresis quite suddenly; no increase of it characteristic of GBS was observed. Typical for GBS are weakness and complete abolition of tendon reflexes in the limbs, which occurred in our patient. In EMG there is a conduction block with scattering of collective muscle action potentials. Electromyography in most cases shows demyelinating changes; only axonal changes are rare. Many cases of GBS in the 
course of SLE have been reported in the literature [7, 8]. There are also GBS forms with primary axonal damage [8] and acute motor axonal neuropathy (AMAN) and acute motor-sensory axonal neuropathy (ASMAN).

In Europe and the United States such complications as AMAN and ASMAN are very rare and they affect only $5 \%$ of patients with GBS [9]. Acute motor axonal neuropathy is a polyneuropathy associated with enteritis Campylobacter jejuni infection and presence of antiganglioside antibodies. Unlike GBS and CIP, tendon reflexes can be preserved in AMAN and later during recovery can be excessive, which was not observed in our patient. Autonomic dysfunctions, such as hypertension, or fluctuations of blood pressure or heart rate, are frequently seen in patients with GBS but are not so common in AMAN [10]. In EMG in AMAN the studies have shown that in the stages in addition to axonal loss, patients have also rapidly reversible conduction block or slowing, in the early phase of the disease [11]. In our EMG, performed quite quickly after the onset of symptoms, these changes were not found.

In addition, Miller Fisher syndrome with ophthalmoplegia, ataxia, areflexia, as well as peripheral facial nerve damage, is distinguished, and various overlap syndromes in which lower cranial nerve damage may also occur, which was not observed in the patient. In patients with axonal GBS antibodies against GM1, GM1b and GD1a infection with C. jejuni was described [12]. This immune reaction against $C$. jejuni leads to the induction of antibodies that can cross-react with gangliosides which are expressed on the peripheral motor axons [13].

Unfortunately, in our case we did not carry out antiGM1 antibody tests. As we know, only $25 \%$ of patients had these antibodies, and after EMG less than $10 \%$ of patients had diagnosed AMAN [14]. In CIP the cerebrospinal fluid protein level is normal, and cranial nerve and autonomic functions are usually preserved; all of which distinguish this disorder from the GBS, but in the described case no cerebrospinal fluid examination was performed due to the lack of the patient's consent. However, the asymmetrical occurrence of symptoms with undisturbed autonomous function and EMG results directed the diagnosis to CIP.

Critical illness myopathy (CIM) and CIP, as well as overlapping of both conditions (critical illness polyneuromyopathy - CIPNM), are the most common cause of neuromuscular weakness in the intensive care setting $[15,16]$. Especially there is a relationship between infections and the development of axonal polyneuropathy symptoms. The clinical course with recurrent septic shock and prolonged respiratory failure points towards CIP. This disease was first described by Bolton et al. [7] in 1984 . It is reported in $25-45 \%$ of cases of critically ill patients [16, 17]. The lower limbs, diaphragm and additional respiratory muscles are mainly affected. Occasional attachment of facial and ocular muscles may also be included in the picture of the disease.

The main independent risk factors for CIP are in particular Gram-negative bacteremia, as well as hyperglycemia and other severe septic states. Female gender and GC therapy, and other factors such as hyperosmolarity, hypalbuminemia, hypoxia, hypothermia, hyper/hypocalcemia, older age, multiple morbidity, damage to the central nervous system such as septic encephalopathy, long duration of organ dysfunction, injury of kidneys, condition after kidney transplantation, parenteral nutrition, and supportive treatment with vasopressor amines are risk factors for CIP development [15-17].

Critical illness polyneuropathy and CIM are complications that manifest as muscle weakness and dependence on mechanical ventilation in intensive care patients. The disease appears after a few days or a longer duration of the critical state and is preceded in most cases by a state of clouding or a state of reduced consciousness, which also occurred in our patient. Polyneuropathy can be diagnosed later when it causes difficulties in weaning the patient from ventilation, even when the underlying general disease has been managed [18, 19].

In addition to prolonging mechanical ventilation and hospitalization, CIP and CIM increase hospital mortality in critically ill patients and cause chronic disability in survivors [15]. Structural changes associated with CIP and CIM include axonal degeneration, loss of muscle myosin, and muscle necrosis $[16,17]$. Acquired sodium channelopathy resulting in reduced excitability of muscle membranes and nerves is a possible mechanism underlying CIP and CIM. Diagnosis of CIP, CIM or combined CIP and CIM is based on clinical, electrophysiological and muscle biopsy tests. In our case, no muscle damage was observed. In EMG the findings are associated with axonal polyneuropathy with loss of amplitudes in both sensory and motor nerve action potentials [20]. These changes are more pronounced in the lower extremities, typically bilaterally, but not necessarily symmetrically, as seen in our patient. About $20 \%$ of patients diagnosed with CIM or CIP have prolonged weakness of sensory changes at the time of discharge [21].

Our patient's neurological condition significantly improved after the treatment and rehabilitation. Early rehabilitation in the intensive care unit can improve the functional status and independence of patients. The course of our patient's axonal polyneuropathy was initially turbulent, but after four months of rehabilitation, the patient was walking, and there was a significant improvement in the neurological condition. The functional state of the patient with a slight paresis of the 
lower extremities and the abolition of tendon reflexes persisted, which suggests sudden damage, which was reversible.

\section{Conclusions}

Critical illness polyneuropathy, often associated with infections, may also occur in a patient with SLE, which will make it difficult to analyze changes in the course of lupus. The overlapping of infection additionally hinders the diagnosis and worsens the prognosis. Neurological diagnostics, including imaging tests of the nervous system and electrophysiological tests, is indicated in all cases of suspected polyneuropathy or myopathy in SLE patients.

After analysis of the literature and observation in the described case, the patient was diagnosed with CIP. The described clinical situation also draws attention to the positive role of early comprehensive rehabilitation including both improving the musculoskeletal system and neurological rehabilitation in patients with autoimmune disease after a critical systemic condition.

The authors declare no conflict of interest.

\section{References}

1. The American College of Rheumatology nomenclature and case definitions for neuropsychiatric lupus syndromes. Arthritis Rheum 1999; 42: 599-608, DOI: 10.1002/1529-0131(199904) 42:4<599::AID-ANR2>3.0.CO;2-F.

2. Shaban A, Leira EC. Neurological complications in patients with systemic lupus erythematosus. Curr Neurol Neurosci Rep 2019; 19: 97, DOI: 10.1007/s11910-019-1012-1.

3. Gao Z, Li X, Peng T, et al. Systemic lupus erythematosus with Guillian-Barre syndrome: A case report and literature review. Medicine (Baltimore) 2018; 97: e11160, DOI: 10.1097/ MD.0000000000011160.

4. López-López CO, Montes Castillo ML, Soto-Fajardo RC, et al. Peripheral neuropathies in rheumatic diseases: More diverse and frequent than expected. A cross-sectional study. Int I Rheum Dis 2020; 23: 226-232, DOI: 10.1111/1756-185X.13755.

5. Victor M, Ropper $\mathrm{AH}$. Neurologia Adamsa i Viktora. $8^{\text {th }}$ ed. Czelej, Lublin 2003: 322-335.

6. Coomes EA, Haghbayan H, Spring J, Mehta S. Fulminant Guillain-Barré syndrome in a patient with systemic lupus erythematosus. BMJ Case Rep 2019; 12: bcr-2018-226634, DOI: 10.1136/bcr-2018-226634.

7. Bolton CF. Evidence of neuromuscular dysfunction in the early stages of the systemic inflammatory response syndrome.
Intensive Care Med 2000; 26: 1179-1180, DOI: 10.1007/ s001340000622.

8. Hughes RAC, Cornblath DR. Guillain-Barré syndrome. Lancet 2005; 366: 1553-1666, DOI: 10.1016/S0140-6736(05)67665-9.

9. Pons-Estel GJ, Ugarte-Gil MF, Alarcón GS. Epidemiology of systemic lupus erythematosus. Expert Rev Clin Immunol 2017; 13: 799-814, DOI: 10.1080/1744666X.2017.1327352.

10. Asahina M, Kuwabara S, Suzuki A, Hattori T. Autonomic function in demyelinating and axonal subtypes of Guillain-Barré syndrome. Acta Neurol Scand 2002; 105: 44-50, DOI: 10.1034/ j.1600-0404.2002.00099.x.

11. Kokubun N, Nishibayashi M, Uncini A, et al. Conduction block in acute motor axonal neuropathy. Brain 2010; 133: 2897-2908, DOI: 10.1093/brain/awq260.

12. Yuki N, Kuwabara S, Koga M, Hirata K. Acute motor axonal neuropathy and acute motor-sensory axonal neuropathy share a common immunological profile. J Neurol Sci 1999; 168: 121-126, DOI: 10.1016/s0022-510x(99)00180-x.

13. Yuki N, Susuki K, Koga M, et al. Carbohydrate mimicry between human ganglioside GM1 and Campylobacter jejuni lipooligosaccharide causes Guillain-Barre syndrome. Proc Natl Acad Sci U S A 2004; 101: 11404-11409, DOI: 10.1073/pnas.0402391101.

14. Tam CC, Rodrigues LC, O’Brien SJ. Guillain-Barré syndrome associated with Campylobacter jejuni infection in England, 2000-2001. Clin Infect Di 2003; 37: 307-310, DOI: 10.1086/ 375845 .

15. Latronico N, Bolton CF. Critical illness polyneuropathy and myopathy: a major cause of muscle weakness and paralysis. Lancet Neurol 2011; 10: 931-941, DOI: 10.1016/S14744422(11)70178-8.

16. Shepherd S, Batra A, Lerner DP. Review of critical illness myopathy and neuropathy. Neurohospitalist 2017; 7: 41-48, DOI: 10.1177/1941874416663279.

17. Zhou C, Wu L, Ni F, et al. Critical illness polyneuropathy and myopathy: a systematic review. Neural Regen Res 2014; 9 : 101-110, DOI: 10.4103/1673-5374.125337.

18. Oud L. Epidemiology and outcomes of sepsis among hospitalizations with systemic lupus erythematosus admitted to the ICU: a population-based cohort study. J Intensive Care 2020; 8: 3, DOI: 10.1186/s40560-019-0424-y.

19. Arce-Salinas CA, Villasenor-Ovies P. Infections and systemic lupus erythematosus. In: Systemic lupus erythematosus. Almoalim H (ed.). IntechOpen 2012: 407-428, DOI: $10.5772 /$ 27509.

20. Lacomis D. Electrophysiology of neuromuscular disorders in critical illness. Muscle Nerve 2013; 47: 452-463, DOI: 10.1002/ mus.23615.

21. Guarneri B, Bertolini G, Latronico N. Long-term outcome in patients with critical illness myopathy or neuropathy: the Italian multicentre CRIMYNE study. J Neurol Neurosurg Psychiatry 2008; 79: 838-841, DOI: 10.1136/jnnp.2007.142430. 